\title{
Down-regulated MYH11 Expression Correlates with Poor Prognosis in Stage II and III Colorectal Cancer
}

\author{
Ren-Jie Wang ${ }^{1,3 \&}$, Peng Wu ${ }^{1,3 \&}$, Guo-Xiang Cai ${ }^{1,3}$, Zhi-Min Wang ${ }^{1,3}$, Ye Xu ${ }^{1,3}$, Jun- \\ Jie Peng ${ }^{1,3}$, Wei-Qi Sheng ${ }^{2,3}$, Hong-Fen Lu$^{2,3}$, San-Jun Cai ${ }^{1,3 *}$
}

\begin{abstract}
The MYH11 gene may be related to cell migration and adhesion, intracellular transport, and signal transduction. However, its relationship with prognosis is still uncertain. The aim of this study was to investigate correlations between MYH11 gene expression and prognosis in 58 patients with stage II and III colorectal cancer. Quantitative real-time polymerase chain reaction was performed in fresh CRC tissues to examine mRNA expression, and immunohistochemistry was performed with paraffin-embedded specimens for protein expression. On univariate analysis, MYH11 expression at both mRNA and protein levels, perineural invasion and lymphovascular invasion were related to disease-free survival $(p<0.05$; log-rank test). Cancers with lower MYH11 expression were more likely to have a poor prognosis. Otherwise, MYH11 expression was unrelated to patient clinicopathological features. On multivariate analysis, low MYH11 expression proved to be an independent adverse prognosticator $(p<0.05)$. These findings show that MYH11 can contribute to predicting prognosis in stage II and III colorectal cancers.
\end{abstract}

Keywords: MYH11 - realtime PCR - immunohistochemistry - prognosis - colorectal cancer

Asian Pac J Cancer Prev, 15 (17), 7223-7228

\section{Introduction}

Colorectal cancer $(\mathrm{CRC})$ is responsible for more than 500, 000 deaths worldwide every year. Stage II and III tumors together represent approximately $70 \%$ of CRC patients. Multidisciplinary treatments induce clinical remission in about $60 \%$ of stage II and III cases. However, $40 \%$ to $50 \%$ of patients will relapse, and most of them will die from secondary diseases (Cserni, 2003; de Gramont et al., 2007). Tumor stage by American Joint Committee on Cancer (AJCC) (Compton et al., 2000) and differentiation are the main prognostic factors used in clinical practice. However, they are not sufficient to evaluate the likelihood of survival. Therefore, biomarkers are urgently needed to predict prognosis and facilitate treatment (Ding et al., 2012; Fang et al., 2012; Lu et al., 2012; Deng et al., 2013; Hu et al., 2013; Li et al., 2013).

Myosin-11 is a protein that in humans is encoded by the MYH11 gene (Matsuoka et al., 1993). It is a major contractile protein, converting chemical energy into mechanical energy through the hydrolysis of ATP. Recently, some researches have shown that myosin has many intracellular functions, including cell migration and adhesion, intracellular transport, locating of organelles and macromolecules and signal transduction and tumor suppression, which indicate that Myosin-11 is more than a contractile protein. Moreover, recent researches have found that Myosins play important roles in cancers (Loikkanen et al., 2009; Cui et al., 2010; Jacobs et al., 2010; Pessina et al., 2010; Yang et al., 2013). However, the clinical significance of Myosin expression in colorectal cancer remains unclear. There is no report concerning the prognostic value of MYH11 in CRC samples, especially in stage II and III samples. In the present study, we investigated MYH11 expression using quantitative real-time polymerase chain reaction (realtime PCR) and immunohistochemistry. And the correlations between MYH11 expression and clinicopathologic features and disease-free survival time were analyzed.

\section{Materials and Methods}

\section{Patients and Sample Collection}

58 stage II/III colorectal cancer patients who received radical surgery in the Department of Colorectal Surgery in Fudan University Shanghai Cancer Center between 2006 and 2008 were studied. The research protocol was approved by the ethics committee in Fudan University Shanghai Cancer Center. Fresh tissues were collected for mRNA examination. Paraffin-embedded colorectal neoplastically changed specimens were collected retrospectively from Department of Pathology in Fudan 
University Shanghai Cancer Center for protein analysis. The characteristics of all patients are shown in Table 1. All specimens were taken from vital cores of histopathogically confirmed cancers at primary curative surgery in patients who did not undergo any chemotherapy or radiotherapy before surgery. Tumor samples were reviewed by 2 experienced pathologists, and tumor stage was assigned on the basis of the system of Cancer Staging Manual by AJCC $7^{\text {th }}$ edition. No stage II but all stage III patients received postoperative chemotherapy (FOLFOX), but no patient received radiotherapy. Disease-free survival was defined as the time elapsed from the date of the initial diagnosis to the appearance of local recurrence or distant metastasis.

\section{RNA analysis by Quantitative Real-time PCR and reverse transcriptase PCR}

Total RNA was isolated with RNAprep Pure Tissue Kit (Tiangen, China) and treated with DNase. According to the manufacturer's instructions, cDNAs were synthesized with QuantScript RT Kit (Tiangen, China). The cDNA was subject to realtime PCR analysis with TaqMan assays from Roche for MYH11 (NM_001040113.1, NM_001040114.1, NM_022844.2, NM_002474.2), and Beta-Actin (NM_001101.3). The realtime PCR reactions were performed using Roche Lightcycler TaqMan master mix according to the manufacturer's instructions. Reactions were performed using ABI Prism 7900HT (Applied Biosystems, USA) utilizing a 384 well block. Thermal cycle conditions consisted of an initial denaturizing incubation at $95^{\circ} \mathrm{C}$ for $10 \mathrm{~min}$, followed by 40 cycles of denaturizing at $95^{\circ} \mathrm{C}$ for $15 \mathrm{sec}$, annealing at $57^{\circ} \mathrm{C}$ for $30 \mathrm{sec}$, and stretching at $72^{\circ} \mathrm{C}$ for $30 \mathrm{sec}$. Data were analyzed using ABI Prism 7900 SDS software (Sequence Detection System 2.0; Applied Biosystems). Data was analyzed by the delta/delta $\mathrm{Ct}$ method in which all target gene MYH11 $\mathrm{Ct}$ values are adjusted to corresponding reference gene $\beta$-Actin Ct levels. All experiments were repeated 3 times.

\section{Immunohistochemistry}

Archival hematoxylin and eosin-stained slides were reviewed by 2 experienced pathologists in accordance with the 2012 World Health Organization classification. A 4-tiered histologic grading system was applied. The TNM stage was evaluated according to Cancer Staging Manual by AJCC 7th edition. The polyclonal goat antihuman MYH11 antibody (ab133567, Abcam, MA, United States) (dilution 1:100) for immunohistochemistry was used. In this study, 4- $\mu$ m sections from archival paraffin blocks were deparaffinized and heated twice for 10 minutes each in a microwave oven $(500 \mathrm{~W})$ before exposure to the first antibody. Immunoperoxidase staining was carried out using the 2-step EnVision method (DAKO, Glostrup, Denmark) according to the manufacturer's instructions and visualized with 3, 3'-diaminobenzidine tetrachloride (Sigma, St Louis, MO).

\section{Statistical analysis}

We used SPSS version 13.0 for Windows (IL, Chicago, Inc) for statistical analysis. MYH11 expression and other related clinicopathologic features were analyzed with prognosis using survival curves. Survival curves were generated by the Kaplan-Meier method, and univariate survival distributions were compared using the logrank test. The $\chi^{2}$ test was used to evaluate the statistical significance of the association of MYH11 expression with the patients' clinicopathologic parameters. The significant factors found in the univariate analysis were put into multivariate analysis, the multivariate Cox proportional hazards model was used for detection of independent prognosticator. The 2-tailed $\mathrm{P}$ value for significance was established at 0.05 .

\section{Results}

MYH11 expression and prognosis (univariate analysis)

We tested the expression of MYH11 gene in both mRNA level and protein level. In mRNA level, we have used quantitative realtime PCR to test the expression of mRNA. All relative expression levels were ranked from low to high, and samples in the first half were defined as "low expression", while samples in the second half were defined as "strong expression". In protein level, cytoplasm and membrane staining were measured for this antibody. Positive cells were counted by 2 pathologists who were blinded to the clinical outcome. We used a 4-tiered scoring system (negative to $3+$ ), which took the percentage of positive cells and staining intensity into account (Fromowitz et al., 1987). We separately interpreted MYH11 1- and 1+ as "low expression" and MYH11 2+ and 3+ as "high expression" (Figure 1). According to this determination, 15 samples (26\%) are defined as high expression, while 43 samples (74\%) are defined as low expression.

In order to study the prognostic value of MYH11 gene, we have followed the patients. The follow-up interval is

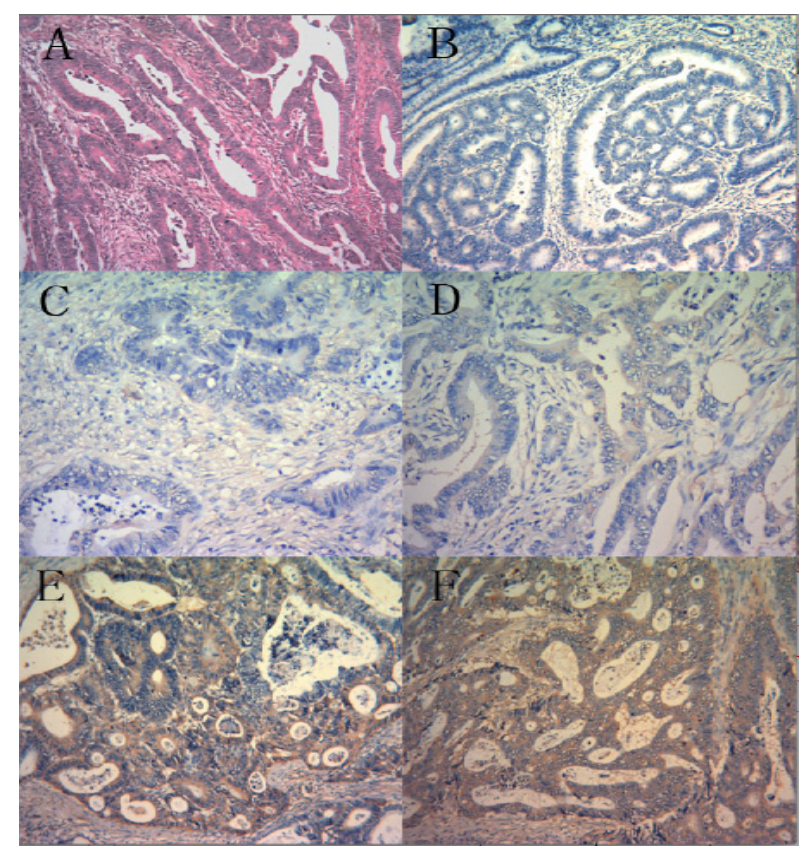

Figure 1. Immunohistochemical Expression of MYH11 in Stage II and III CRC Tissues. Hematoxylin and eosin stain $(A ; \times 100)$. Negative control sample omitting primary antibody $(\mathrm{B} ; \times 100)$. Examples of immunostaining of MYH11 at low expression $(C, D)$ and strong expression $(E, F)$ levels $(\times 100)$ 
from 3 months to 75 months, the median time is 55 months. The follow-up data was analyzed with the expression of MYH11. In mRNA level, expression of MYH11 is Table 1. Patients' Clinicopathologic Features and Disease-Free Survival

\begin{tabular}{|c|c|c|}
\hline Variables & $\mathrm{N}$ & $\mathrm{P}(\log$-rank test $)$ \\
\hline$\overline{\text { Age }}$ & & 0.091 \\
\hline$<75$ & 54 & \\
\hline$\geq 75$ & 4 & \\
\hline Sex & & 0.108 \\
\hline Male & 34 & \\
\hline Female & 24 & \\
\hline Differentiation & & 0.717 \\
\hline Well-moderate & 42 & \\
\hline Poor & 16 & \\
\hline Location & & 0.827 \\
\hline Rectum & 32 & \\
\hline Colon & 26 & \\
\hline Invasive depth & & 0.147 \\
\hline $\mathrm{T} 1-\mathrm{T} 2$ & 1 & \\
\hline T3-T4 & 57 & \\
\hline Lymphnode metastasis & & 0.447 \\
\hline Yes & 35 & \\
\hline No & 23 & \\
\hline Perineural invasion & & 0.021 \\
\hline Yes & 14 & \\
\hline No & 44 & \\
\hline Lymphovascular invasion & & 0.023 \\
\hline Yes & 19 & \\
\hline No & 39 & \\
\hline MYH11 mRNA expression & & 0.04 \\
\hline High & 29 & \\
\hline Low & 29 & \\
\hline MYH11 protein expression & & 0.005 \\
\hline High & 15 & \\
\hline Low & 43 & \\
\hline
\end{tabular}

Table 2. Patients' Clinicopathologic Features and MYH11 Protein Expression

\begin{tabular}{|c|c|c|c|c|}
\hline Variables & $\mathrm{N}(\%)$ & $\begin{array}{l}\text { MYH11 ex } \\
\text { high }(n=)\end{array}$ & $\begin{array}{l}\text { pression } \\
\text { low }(\mathrm{n}=)\end{array}$ & $\mathrm{P}\left(\chi^{2}\right)$ \\
\hline \multicolumn{2}{|l|}{ Age } & \multicolumn{3}{|c|}{0.564} \\
\hline$<75$ & $54(93)$ & 15 & 39 & \\
\hline$\geq 75$ & $4(7)$ & 0 & 4 & \\
\hline \multicolumn{2}{|l|}{ Sex } & \multicolumn{3}{|c|}{0.9} \\
\hline Male & $34(59)$ & 9 & 25 & \\
\hline Female & $24(41)$ & 6 & 18 & \\
\hline \multicolumn{4}{|l|}{ Differentiation } & \multirow[t]{3}{*}{1} \\
\hline Well-moderate & $42(72)$ & 11 & 31 & \\
\hline Poor & $16(28)$ & 4 & 12 & \\
\hline \multicolumn{4}{|l|}{ Location } & \multirow[t]{3}{*}{0.17} \\
\hline Rectum & $32(55)$ & 6 & 26 & \\
\hline Colon & $26(45)$ & 9 & 17 & \\
\hline \multicolumn{4}{|l|}{ Invasive depth } & \multirow[t]{3}{*}{1} \\
\hline $\mathrm{T} 1-\mathrm{T} 2$ & $1(2)$ & 0 & 1 & \\
\hline T3-T4 & $57(98)$ & 15 & 42 & \\
\hline \multicolumn{4}{|c|}{ Lymphnode metastasis } & \multirow[t]{3}{*}{0.208} \\
\hline Yes & $35(60)$ & 7 & 28 & \\
\hline No & $23(40)$ & 8 & 15 & \\
\hline \multicolumn{4}{|l|}{ Perineural invasion } & \multirow[t]{3}{*}{1} \\
\hline Yes & $14(24)$ & 3 & 11 & \\
\hline No & $44(76)$ & 12 & 32 & \\
\hline \multicolumn{4}{|c|}{ Lymphovascular invasion } & \multirow[t]{3}{*}{0.183} \\
\hline Yes & $19(33)$ & 7 & 12 & \\
\hline No & $39(67)$ & 8 & 31 & \\
\hline
\end{tabular}

related to patients' disease-free survivals (DFS), the 5 -year disease-free survival rate is $44.8 \%$ in the "low expression" group and $75.9 \%$ in the "high expression" group. In protein level, expression of MYH11 is also related to patients' DFS, the 5-year disease free survival rate is $48.8 \%$ in the "low expression" group and $93.3 \%$ in the "strong expression" group (Table 1, Figure 2).

\section{Related clinicopathologic features and prognosis}

According to the follow-up data and patients' clinicopathologic features, we found that patients' gender, age, tumor differentiation, location, invasive depth, lymphnode metastasis were unrelated to prognosis, while perineural invasion and lymphovascular invasion affact patients' disease free survival (Table 1, Figure 2).

Table 3. Multivariate Analysis of Disease-Free Survival in Stage II /III Colorectal Cancer

\begin{tabular}{lllll}
\multicolumn{1}{c}{ Factor } & & & \multicolumn{2}{c}{$95 \%$ CI } \\
\cline { 4 - 5 } & $H R$ & $P$ & Lower & Upper \\
\hline MYH11 protein expression & 0.113 & 0.035 & 0.015 & 0.86 \\
Perineural invasion & 1.207 & 0.72 & 0.431 & 3.381 \\
Lymphovascular invasion & 2.009 & 0.171 & 0.741 & 5.448 \\
\hline *Abbreviation: CI, confidence interval. & & &
\end{tabular}

A
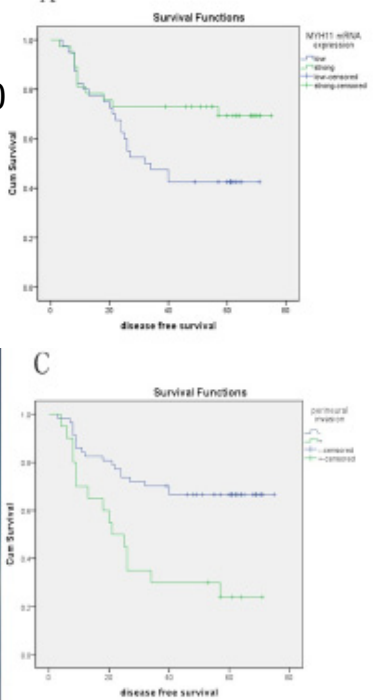

B

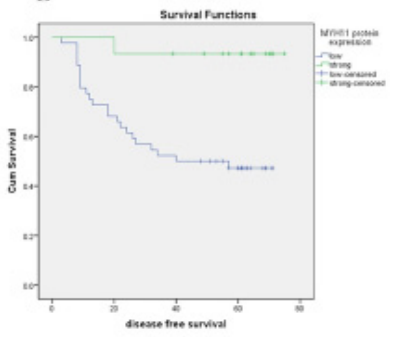

D

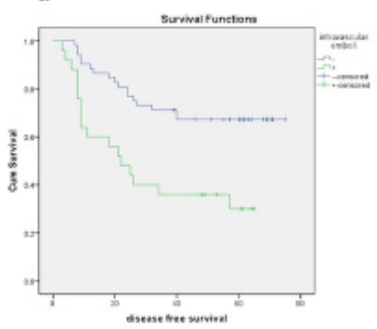

Figure 2. Clinicopathologic features and diseasefree survival. A:Kaplan-Meier estimated survival rates according to MYH11 mRNA expression. Patients with low MYH11 mRNA expression $(n=29)$ showed a significantly poorer prognosis than those with high MYH11 mRNA expression $(\mathrm{n}=29 ; p<0.05$; log-rank test). B: Kaplan-Meier estimated survival rates according to MYH11 protein expression. Patients with low MYH11 protein expression $(n=43)$ showed a significantly poorer prognosis than those with high MYH11 protein expression $(\mathrm{n}=15 ; p<0.05 ; \log$-rank test). C: Kaplan-Meier estimated survival rates according to perineural invasion. Patients with perineural invasion $(n=14)$ showed a significantly poorer prognosis than those without perineural invasion ( $\mathrm{n}=44 ; p<0.05$; log-rank test). D: Kaplan-Meier estimated survival rates according to lymphovascular invasion. Patients with lymphovascular invasion $(n=19)$ showed a significantly poorer prognosis than those without lymphovascular invasion $(\mathrm{n}=39 ; p<0.05$; log-rank test) 
The relationship between MYH11 gene expression and clinicopathologic features in stage II and III colorectal cancer

In order to find the potential function of MYH11 gene in stage II and III colorectal cancer, we also use $\chi^{2}$ test to compare the MYH11 gene expression and patients' clinicopathologic features, and we found that there is no relation between MYH11 gene expression and patients' clinicopathologic features (Table 2).

\section{MYH11 expression and prognosis (multivariate analysis)}

According to the results of univariate analysis, perineural invasion, lymphovascular invasion and expression of MYH11 were put into multivarient analysis. The Cox proportional hazards model shows that expression of MYH11 is an independent predictor for prognosis (Table 3).

\section{Discussion}

Since the morbidity is rising, more and more researchers from different regions are paying close attention on colorectal cancer (Atrkar-Roushan et al., 2013; Gao et al., 2013; Heidarnia et al., 2013; Lohsiriwat et al., 2013; Madrigal-Bujaidar et al., 2013; Saeed et al., 2013). Among them, finding biomarkers for prognosis has long been a hotspot (Huang et al., 2012; Qian et al., 2012; Zhang et al., 2012; Chen et al., 2013; Cui et al., 2013; Dong et al., 2013; Sisik et al., 2013; Wu et al., 2013). Myosins have a well-characterized biological function in using the energy of ATP hydrolysis to move actin filaments to produce muscle force. For so long, the researches about MYH11 have been concentrated on acute myeloid leukemia (AML), because MYH11 has been involved in the composition of fusion gene $\mathrm{CBF} \beta$ MYH11, and CBF $\beta$ will generally form an intranuclear transcriptionally active complex together with RUNX1, so the fusion gene disturb the function of $\mathrm{CBF} \beta$, which influences hematopoiesis (Goyama and Mulloy, 2011). However, recently myosins are implicated also in a variety of other cellular function and many of which are relevant for cancer formation (Krendel and Mooseker, 2005). So here we have extended the analysis of the role of myosin in colorectal cancer patients.

In our study, we have found that the expression of MYH11 affects patients' disease-free survivals (DFS) in both fresh/paraffin embedded tissues, patients with lower MYH11 expression is likely to have lower 5-year disease free survival rate. Because the expression information from the paraffin block is relatively reliable, we analyzed the relations between MYH11 protein expression in paraffin-embedded specimens and the patients' clinicopathologic characteristics. No correlation was found between MYH11 expression and patients' age, sex, differentiation, location, invasive depth, lymph node metastasis, perineural invasion, or lymphovascular invasion. Moreover, the multivariate analysis showed that the expression of MYH11 was an independent prognosticator. Thus, we confirmed the role of MYH11 in the prognosis of colorectal cancer.

In survival analysis, it is showed that there was no significant difference between stage II and III group (with/ without lymph node metastasis). Since previous research has reported that combined chemotherapy may decrease the risk of recurrence/metastasis by approximately $50 \%$ and gain an additional $20 \%$ absolute benefit at 5 years compared with surgery alone (Aschele et al., 2009), the reason could be explained that chemotherapy prolonged the disease-free survival time in stage III patients. On the other hand, perineural invasion and lymphovascular invasion were shown to be associated with disease free survival together with MYH11 expression, these results coincide with the experimental findings in the previous published papers (Krasna et al., 1988; Liebig et al., 2009).

Our study revealed that low expressers of MYH11 exhibited a poor prognosis trend, with an extremely high risk of recurrence/metastasis $(\mathrm{HR}, 0.113 ; \mathrm{P}<0.05)$. Although the exact function of MYH11 in tumorigenesis and metastasis remains still unclear, there is evidence showing that myosin motor proteins play crucial roles in the events leading to motility at leading edge of migrating cells (Chibalina et al., 2009). So, MYH11 is considered more than a muscular contraction related gene, attention has been paid on its function in cancer: Seitz et al (2006) reported that in breast cancer, MYH11 highly expressed in the hybrids but down-regulated in the tumors and metastases which were confirmed by RT-PCR and microarray analysis. Lu et al (2010) also found that in both human and rodents, MYH11 was commonly downregulated which has conserved functions to the bladder carcinogenesis. There have also been some papers about the function of MYH11 in colorectal cancer. Alhopuro et al (2008) reported that MYH11 mutations appear to contribute to human intestinal neoplasia and unregulated MYH11 may affect the cellular energy balance or disturb cell lineage decisions in tumor progenitor cells. In the microsatellite stable (MSS) hereditary nonpolyposis colorectal cancer (HNPCC), Lee et al (2008) found that MYH11 gene expresses differentially compared with the adjacent pathologically normal tissues. Furthermore, Vickaryous et al (2008) examined adenomas and cancers from hereditary non-polyposis colorectal cancer (HNPCC) patients for the presence of frameshift mutations in the smooth-muscle myosin gene, and found that mutations in MYH11 occur more frequently in cancers than adenomas and was dependent on microsatellite instability. After performing whole-genome sequencing of the tumor, targeted whole-exome sequencing of tumor and normal DNA, and transcriptome sequencing (RNA-Seq) of the tumor to identify potentially informative mutations in a clinically relevant time frame, Roychowdhury et al (2011) identified somatic point mutations of MYH11 in patients having metastatic colorectal cancer. As a result, although there is yet no complete description of the molecular mechanism by which MYH11 induces poor prognosis. We can believe that MYH11 does play an important role in cell death or survival signals emanating from the microenvironment at the secondary site.

Since the most important finding of the present study was that low expression of MYH11 in colorectal cancer patients was significantly associated with poor prognosis, we anticipate that this finding has implications for the 
management of patients with colorectal cancer. First, some stage II cases could be considered for adjuvant therapy, although the role of adjuvant chemotherapy in these patients is still unclear (De Dosso et al., 2009). To select high-risk stage II disease and maximize the benefits of adjuvant therapy, an independent prognostic marker could be helpful in identifying aggressive phenotypes within stage II colorectal cancer. Second, specific surveillance may be applied in both stage II and III colorectal cancer patients exhibiting low expression of MYH11 in order that early recurrence/metastasis can be found early and treated properly.

Of course, there are still some limitations in our research. First, it is a retrospective study, some cases are rejected artificially because of data incompletion or sample imperfection. Second, the sample size still needs to be enlarged in order to verify the present results. Finally, in the further research, in vitro test is necessary to be applied for studying the detailed pathway and regulation of MYH11 in colorectal cancer.

In conclusion, low expression of MYH11 can predict poor prognosis in stage II and III colorectal cancer. The application of MYH11 will help to differentiate patients with high risks, so as to make the benefits of chemotherapy large.

\section{Acknowledgements}

This research is supported by the grant of the National Natural Science Foundation of China (No.81001055), Shanghai Pujiang Program (No.13PJD008) and the grant of the China Medical Foundation (No.201302). We wish to thank all of the participants in this study for their contributions. We greatly appreciate the efforts of Wencui Huang and her colleagues in the Department of Colorectal Surgery, Fudan University Shanghai Cancer Center and their excellent work in sample collection.

\section{References}

Alhopuro P, Phichith D, Tuupanen S, et al (2008). Unregulated smooth-muscle myosin in human intestinal neoplasia. Proc Natl Acad Sci USA, 105, 5513-8.

Aschele C, Bergamo F, Lonardi S (2009). Chemotherapy for operable and advanced colorectal cancer. Cancer Treat Rev, 35, 509-16.

Atrkar-Roushan Z, Kazemnejad A, Mansour-Ghanaei F, et al (2013). Trend analysis of gastrointestinal cancer incidences in Guilan province: comparing rates over 15 years. Asian Pac J Cancer Prev, 14, 7587-93.

Chen Q, Xia HW, Ge XJ, et al (2013). Serum miR-19a predicts resistance to FOLFOX chemotherapy in advanced colorectal cancer cases. Asian Pac J Cancer Prev, 14, 7421-6.

Chibalina MV, Puri C, Kendrick-Jones J, et al (2009). Potential roles of myosin VI in cell motility. Biochem Soc T, 37, 966-70.

Compton C, Fenoglio-Preiser CM, Pettigrew N, et al (2000). American joint committee on cancer prognostic factors consensus conference. Cancer, 88, 1739-57.

Cserni G (2003). Nodal staging of colorectal carcinomas and sentinel nodes. J Clin Pathol, 56, 327-35.

Cui WJ, Liu Y, Zhou XL, et al (2010). Myosin light chain kinase is responsible for high proliferative ability of breast cancer cells via anti-apoptosis involving p38 pathway. Acta Pharmacol Sin, 31, 725-32.

Cui YL, Li HK, Zhou HY, et al (2013). Correlations of tumorassociated macrophage subtypes with liver metastases of colorectal cancer. Asian Pac J Cancer Prev, 14, 1003-7.

De Dosso S, Sessa C, Saletti P (2009). Adjuvant therapy for colon cancer: present and perspectives. Cancer Treat Rev, 35, 160-6.

de Gramont A, Tournigand C, André T, et al Adjuvant therapy for stage II and III colorectal cancer. Semin Oncol, 2007, 37.

Deng BG, Yao JH, Liu QY, et al (2013). Comparative serum proteomic analysis of serum diagnosis proteins of colorectal cancer based on magnetic bead separation and maldi-tof mass spectrometry. Asian Pac J Cancer Prev, 14, 6069-75.

Ding YL, Wang QS, Zhao WM, et al (2012). Expression of smoothened protein in colon cancer and its prognostic value for postoperative liver metastasis. Asian Pac J Cancer Prev, 13, 4001-5.

Dong H, Tang J, Li LH, et al (2013). Serum carbohydrate antigen 19-9 as an indicator of liver metastasis in colorectal carcinoma cases. Asian Pac J Cancer Prev, 14, 909-13.

Fang WJ, Zheng Y, Wu LM, et al (2012). Genome-wide analysis of aberrant DNA methylation for identification of potential biomarkers in colorectal cancer patients. Asian Pac J Cancer Prev, 13, 1917-21.

Fromowitz FB, Viola MV, Chao S, et al (1987). ras p21 expression in the progression of breast cancer. Hum Pathol, 18, 1268-75.

Gao CM, Ding JH, Li SP, et al (2013). Polymorphisms in XRCC1 gene, alcohol drinking, and risk of colorectal cancer: a case-control study in jiangsu province of China. Asian Pac J Cancer Prev, 14, 6613-8.

Goyama S, Mulloy JC (2011). Molecular pathogenesis of core binding factor leukemia: current knowledge and future prospects. Int J Hematol, 94, 126-33.

Heidarnia MA, Monfared ED, Akbari ME, et al (2013). Social determinants of health and 5-year survival of colorectal cancer. Asian Pac J Cancer Prev, 14, 5111-6.

$\mathrm{Hu}$ Y, Wang JL, Tao HT, et al (2013). Expression and significance of TSGF, CEA and AFP in patients before and after radical surgery for colon cancer. Asian Pac J Cancer Prev, 14, 3877-80.

Huang GL, Guo HQ, Yang F, et al (2012). Activating transcription factor 1 is a prognostic marker of colorectal cancer. Asian Pac J Cancer Prev, 13, 1053-7.

Jacobs K, Van Gele M, Forsyth R, et al (2010). P-cadherin counteracts myosin II-B function: implications in melanoma progression. Mol Cancer, 9, 255.

Krasna MJ, Flancbaum L, Cody RP, et al (1988). Vascular and neural invasion in colorectal carcinoma. Incidence and prognostic significance. Cancer, 61, 1018-23.

Krendel M, Mooseker MS (2005). Myosins: tails (and heads) of functional diversity. Physiology, 20, 239-51.

Lee WS, Seo G, Shin HJ, et al (2008). Identification of differentially expressed genes in microsatellite stable HNPCC and sporadic colon cancer. J Surg Res, 144, 29-35.

Li ZT, Zhang L, Gao XZ, et al (2013). Expression and significance of the Wip1 proto-oncogene in colorectal cancer. Asian Pac J Cancer Prev, 14, 1975-9.

Liebig C, Ayala G, Wilks JA, et al (2009). Perineural invasion in cancer: a review of the literature. Cancer, 115, 3379-91.

Lohsiriwat V, Anubhonganant W, Prapasrivorakul S, et al (2013). Outcomes of local excision for early rectal cancer: a 6-year experience from the largest university hospital in Thailand. Asian Pac J Cancer Prev, 14, 5141-4.

Loikkanen I, Toljamo K, Hirvikoski P, et al (2009). Myosin 
VI is a modulator of androgen-dependent gene expression. Oncol Rep, 22, 991-5.

Lu P, Liu R, Ma EM, et al (2012). Functional analysis of B7$\mathrm{H} 3$ in colonic carcinoma cells. Asian Pac J Cancer Prev, 13, 3899-903.

Lu Y, Liu P, Wen W, et al (2010). Cross-species comparison of orthologous gene expression in human bladder cancer and carcinogen-induced rodent models. Am J Transl Res, 3, 8-27.

Madrigal-Bujaidar E, Martino Roaro L, Garcia-Aguirre K, et al (2013). Grapefruit juice suppresses azoxymethane-induced colon aberrant crypt formation and induces antioxidant capacity in mice. Asian Pac J Cancer Prev, 14, 6851-6.

Matsuoka R, Yoshida MC, Furutani Y, et al (1993). Human smooth muscle myosin heavy chain gene mapped to chromosomal region 16q12. Am J Med Genet, 46, 61-7.

Pessina P, Conti V, Pacelli F, et al (2010). Skeletal muscle of gastric cancer patients expresses genes involved in muscle regeneration. Oncol Rep, 24, 741-5.

Qian LY, Li P, Li XR, et al (2012). Multivariate analysis of molecular indicators for postoperative liver metastasis in colorectal cancer cases. Asian Pac J Cancer Prev, 13, 3967-71.

Roychowdhury S, Iyer MK, Robinson DR, et al (2011). Personalized oncology through integrative high-throughput sequencing: a pilot study. Sci Transl Med, 3, 111ra21.

Saeed HM, Alanazi MS, Parine NR, et al (2013). Matrix metalloproteinase-2 (-1306 c $>$ t) promoter polymorphism and risk of colorectal cancer in the Saudi population. Asian Pac J Cancer Prev, 14, 6025-30.

Seitz S, Korsching E, Weimer J, et al (2006). Genetic background of different cancer cell lines influences the gene set involved in chromosome 8 mediated breast tumor suppression. Gene Chromosome Canc, 45, 612-27.

Sisik A, Kaya M, Bas G, et al (2013). CEA and CA 19-9 are still valuable markers for the prognosis of colorectal and gastric cancer patients. Asian Pac J Cancer Prev, 14, 4289-94.

Vickaryous N, Polanco-Echeverry G, Morrow S, et al (2008). Smooth-muscle myosin mutations in hereditary nonpolyposis colorectal cancer syndrome. Brit J Cancer, 99, 1726-8.

Wu HW, Gao LD, Wei GH (2013). hMSH2 and nm23 expression in sporadic colorectal cancer and its clinical significance. Asian Pac J Cancer Prev, 14, 1995-8.

Yang L, Huang XE, Xu L, et al (2013). Role of MYH polymorphisms in sporadic colorectal cancer in China: a case-control, population-based study. Asian Pac J Cancer Prev, 14, 6403-9.

Zhang YY, Chen B, Ding YQ (2012). Metastasis-associated factors facilitating the progression of colorectal cancer. Asian Pac J Cancer Prev, 13, 2437-44. 